\title{
Bevacizumab Combined with Docetaxel or Paclitaxel as First-line Treatment of HER2-negative Metastatic Breast Cancer
}

\author{
LEENA TIAINEN $^{1,2}$, MINNA TANNER $^{1,2}$, OUTI LAHDENPER ${ }^{3}{ }^{3}$ PIA VIHINEN ${ }^{3}$, \\ ARJA JUKKOLA ${ }^{4}$, PEETER KARIHTALA ${ }^{4}$, NIINA PAUNU ${ }^{2}$, \\ TEPPO HUTTUNEN ${ }^{5}$ and PIRKKO-LIISA KELLOKUMPU-LEHTINEN ${ }^{1,2}$ \\ ${ }^{1}$ Department of Oncology, School of Medicine, University of Tampere, Tampere, Finland; \\ ${ }^{2}$ Department of Oncology, Tampere University Hospital, Tampere, Finland; \\ ${ }^{3}$ Department of Oncology, Turku University Central Hospital, Turku, Finland, \\ ${ }^{4}$ Department of Oncology and Radiotherapy, Medical Research Center Oulu, \\ Oulu University Hospital and University of Oulu, Oulu, Finland; \\ ${ }^{5}$ Oy 4Pharma Ltd, Turku, Finland
}

\begin{abstract}
Aim: The study evaluated the efficacy of bevacizumab combined with a taxane-based treatment for advanced breast cancer. Patients and Methods: In this nonrandomized phase II study 65 patients received $10 \mathrm{mg} / \mathrm{kg}$ bevacizumab i.v. (days 1 and $15, q 4 w)$ plus either $50 \mathrm{mg} / \mathrm{m}^{2}$ docetaxel (days 1 and $15, q 4 w$ ) or $90 \mathrm{mg} / \mathrm{m}^{2}$ paclitaxel (days $1,8$ and $15, q 4 w)$ i.v. until disease progression, maximal response, unacceptable toxicity or the withdrawal of consent. Patients without progression continued bevacizumab at $15 \mathrm{mg} / \mathrm{kg}$ i.v. (q3w) alone, or with endocrine therapy. (NCT00979641). Results: Progression-free survival was 11.3 months (95\% confidence interval=9.7-16.0 months) and overall survival was 35.1 months (95\% confidence interval=22.2-50.3 months). More than half of the patients (62\%) responded at least partially. Bevacizumab-related serious adverse events occurred in $10.8 \%$ patients and one patient died because of gastrointestinal perforation. Conclusion: Treating advanced breast cancer with a bevacizumab-containing regimen as the first-line cytotoxic treatment resulted in excellent response rates and long survival.
\end{abstract}

Metastatic breast cancer remains an incurable disease $(1,2)$. In Finland, nearly 5,000 patients are diagnosed with invasive

This article is freely accessible online.

Correspondence to: Leena Tiainen, Department of Oncology, Tampere University Hospital, Teiskontie 35, 33521 Tampere, Finland. Tel: +358 331163130, Fax: +358 331163019, e-mail: leena.tiainen@pshp.fi

Key Words: Advanced breast cancer, phase II study, bevacizumab, taxanes, first-line chemotherapy, maintenance therapy. breast cancer every year, and the incidence has increased steadily over the past decades. The Finnish cancer registry data from 2014 shows that 815 women died of metastatic breast cancer, which was the most common cause of cancer death in women (3). In the CONCORD-2 study, a central analysis of population-based registry data worldwide for cancer survival was conducted, and the results were published in The Lancet in November 2014. The study reported that the treatment results of breast cancer in Finland are among the best in the world. The 5-year-survival rate of patients with breast cancer in Finland was 86.8\% [95\% confidence interval $(\mathrm{CI})=85.9-87.7 \%)$ from 2005-2009, and was the highest in Northern Europe (4). However, new treatment options for advanced human epidermal growth factor receptor 2 (HER2)-negative disease are rare, and the overall survival benefit observed in these patients is modest $(5,6)$. For this reason, advanced HER2-negative breast cancer is a treatment challenge worldwide.

Bevacizumab is a recombinant humanized monoclonal antibody that inhibits vascular endothelial cell proliferation by blocking the binding of vascular endothelial growth factor A (VEGFA) to its receptor, therefore inhibiting tumor angiogenesis (7). Bevacizumab improves the outcomes of cytotoxic treatment in many metastatic malignancies, including colorectal, kidney, lung and ovarian cancer (8-11). There has been much debate about the status of bevacizumab treatment in metastatic breast cancer. Currently, the European Medicines Agency has only approved bevacizumab when combined with paclitaxel or capecitabine in a first or second-line setting (http://www.e ma.europa.eu/ema/). In 2011, the US Food and Drug Administration revoked its accelerated approval of a breast cancer indication for bevacizumab due to the lack of a benefit in breast cancer overall survival and, in addition, due to the potentially life-threatening side-effects (http://www.fda.gov/). 
In locally advanced and metastatic breast cancer, taxanebased treatment (docetaxel or paclitaxel), either in combination with another agent or as single-agent, therapy is considered one of the most effective choices for first-line treatment $(5,12)$, when cytotoxic treatment is needed. Combining bevacizumab with chemotherapy has been studied in certain phase III studies (13-18). Most of these studies investigated the benefit of bevacizumab combined with a taxane. Furthermore, other chemotherapy regimens have been explored, including capecitabine, anthracycline, vinorelbine and gemcitabine. Adding bevacizumab has led to higher response rates and longer progression-free survival (PFS) throughout the trials, but no significant differences in overall survival (OS) have yet been observed.

In addition to chemotherapy options, bevacizumab can also be combined with endocrine therapy, and the effect may be synergistic. Intracellular VEGF and estrogen signaling pathways cross at several points, and it can be hypothesized that adding bevacizumab to hormonal treatment might delay the development of endocrine therapy resistance $(19,20)$. In hormone receptor-positive advanced breast cancer, endocrine treatment with either an anti-estrogen or an aromatase inhibitor is a keystone of the treatment (5). It is used in metastatic breast cancer in biologically non-aggressive forms of the disease and in more aggressive forms after a maximal chemotherapy response has been achieved (5). For the firstline therapy of advanced breast cancer, an aromatase inhibitor combined with bevacizumab was investigated in a phase III LEA trial (21). Similarly, as reported in chemotherapy trials, the endocrine therapy-bevacizumab combination resulted in higher response rates but failed to demonstrate statistically significant improvements in both PFS and OS compared to endocrine-therapy alone.

This study aimed to investigate whether bevacizumab combined with either docetaxel or paclitaxel is a feasible choice for first-line therapy in metastatic breast cancer. The study also evaluated if using bevacizumab maintenance therapy with an endocrine therapy would have synergistic effects.

\section{Patients and Methods}

Patients. We screened and treated 65 patients at three study centers in Finland: Tampere, Oulu and Turku University hospitals. The study was initiated in May 2009 and data closure took place in April 2015. The median follow-up time was 24.1 months (range=1.6-66.3 months). Pre- and postmenopausal women were eligible if they had histologically or cytologically confirmed HER2-negative metastatic adenocarcinoma of the breast and were considered as candidates for taxane treatment. Patients were not allowed any prior chemotherapy for advanced disease but could have been treated with (neo)adjuvant chemotherapy if the disease-free interval was at least 6 months. Previous endocrine therapy for advanced disease was allowed. Both measurable and non-measurable (bone-only) diseases were eligible. Good performance status was required [Eastern Cooperative Oncology
Group (ECOG) performance status 0-2]. Additional inclusion criteria included adequate hematological, renal and hepatic functions.

Patients were excluded if they had history of central nervous system metastases or pre-existing peripheral neuropathy at least grade 2 by National Cancer Institute Common Toxicity Criteria (NCI-CTC), version 3.0 (22). Additionally, circumstances that could increase the serious adverse events associated with bevacizumab were excluded, such as major surgery within the previous month, minor surgery within the last 24 hours prior to bevacizumab initiation, the use of anticoagulants or thrombolytic agents, a history of bleeding diathesis or coagulopathy, uncontrolled hypertension, clinically significant cardiovascular disease, a non-healing wound, an active peptic ulcer or bone fracture, a history of abdominal fistula, and a gastrointestinal perforation or intra-abdominal abscess within 6 months of enrollment. Furthermore, patients with a history of other malignancies were excluded.

The study protocol was approved by the Ethics Committee of Tampere University Hospital (R08142M) and the trial identifier is NCT00979641. Written informed consent was obtained from all patients included in the study.

Treatment. In part I of the treatment, the patients received taxane therapy intravenously (i.v.; $50 \mathrm{mg} / \mathrm{m}^{2}$ docetaxel on days 1 and 15 or $90 \mathrm{mg} / \mathrm{m}^{2}$ paclitaxel on days 1,8 and 15 ) and $10 \mathrm{mg} / \mathrm{kg}$ bevacizumab i.v. on days 1 and 15 on a treatment cycle of 28 days. Treatment was continued until the maximal response, progressive disease, unacceptable toxicities necessitating the termination of taxane treatment or the patient's refusal. The maximal response was defined as an achieved response (a complete response (CR) or a partial response PR) that was the same between two response evaluations, or stable disease (SD) for more than 6 months. The study was initiated with the docetaxel-bevacizumab combination. After the negative results from the AVADO trial (14) were published, an amendment to the study protocol was made and the following enrolled patients were treated with a combination of paclitaxel and bevacizumab. In part II of treatment, after taxane treatment was discontinued, the responding patients continued to receive $15 \mathrm{mg} / \mathrm{kg}$ bevacizumab intravenously on day $1 \mathrm{q} 21$ days. In hormone receptorpositive patients, an endocrine therapy according to the investigator's choice was added to bevacizumab. This second part of the treatment was given until disease progression, unacceptable treatment-related toxicities or the withdrawal of the patient's consent. The study scheme is presented in Figure 1.

After disease progression, the continuation of bevacizumab with a second-line therapy was optional. The preferred chemotherapy option was capecitabine or the investigator's choice. Capecitabine was administered at a dose $1000 \mathrm{mg} / \mathrm{m}^{2}$ twice-daily per os given on days 1-14 of a 3 -week cycle.

Dose modifications, toxicity and response evaluations. The dosing of bevacizumab was not modified during the study. In case of grade 34 bevacizumab-related toxicity, bevacizumab was either temporarily or permanently suspended. If bevacizumab was permanently discontinued but chemotherapy not interrupted, the patient entered the follow-up phase of the study. The bevacizumab-related toxicities were monitored closely and specific treatment algorithms were made for hypertension, proteinuria, thromboembolic events, hemorrhage, gastrointestinal perforations and impaired wound-healing. The dose of the taxane was allowed to be reduced according to each clinic's standards of care in the case of taxane-related toxicity. Toxic effects 


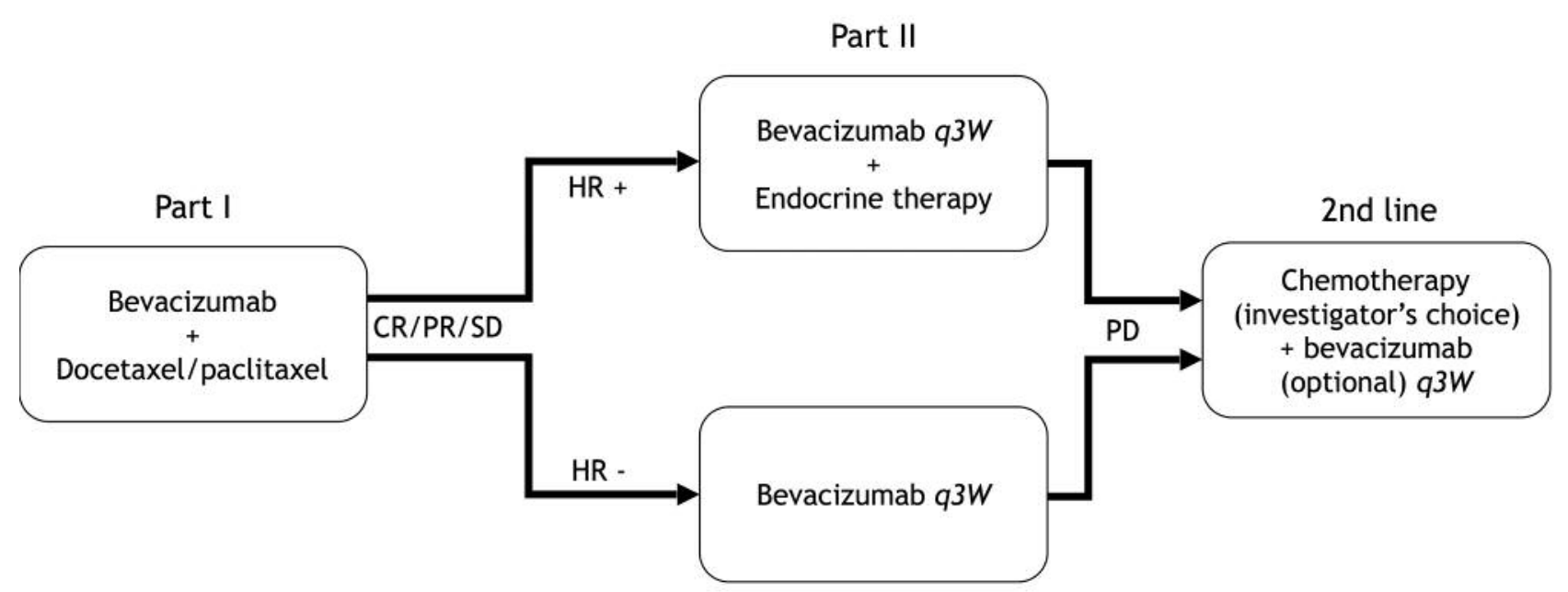

Figure 1. Study scheme. CR: Complete response, PR: partial response, SD: stable disease, HR: hormone receptor, q3W: every 3 weeks, PD: progressive disease.

were graded according to the NCI-CTC, version 3.0 (22). For secondline capecitabine, dose modifications were made according to the investigator's assessment. In patients with moderate renal impairment, the dose of capecitabine was reduced by $25 \%$.

Tumor assessment was performed every 12 weeks until progression, according to the Response Evaluation Criteria in Solid Tumors (RECIST), version 1.1 (23). All patients were followed-up every 6 months for an evaluation of their status and for survival by following the patient records.

Statistical analysis. The primary endpoint of the study was PFS in the first-line treatment setting and it was calculated from the date of treatment initiation to the date of investigator-assessed disease progression according to the RECIST criteria (23) or to the date of patient death. Secondary end-points were safety, the response rate and OS. Adverse events are displayed in standard frequency tables. The proportions of patients with $\mathrm{CR}, \mathrm{PR}, \mathrm{SD}$ and progressive disease (PD) as the best response were tabulated for each part of the treatment. OS was calculated from the date of treatment initiation to the date of death due to any cause. The median PFS and OS were calculated according to the Kaplan-Meier method. The analysis of PFS and OS included the stratification variable taxane choice (docetaxel-bevacizumab or paclitaxel-bevacizumab) and hormone receptor status. The Kaplan-Meier estimates obtained from the model were compared with the historical control group (14).

A total of 65 patients were expected to enter the study. This number would provide a probability of $80 \%$ for detecting a difference corresponding to a ratio of 1.34 between this study group and historical control group (equal to PFS of 10.7 months versus 8 months). The basis of the assumptions was that the accrual period was 18 months, the follow-up period was 36 months and the median PFS of the historical control group was 8.2 months in a series of 241 patients (14).

\section{Results}

Patient baseline characteristics. Between May 2009 and October 2013, 65 patients were enrolled. The baseline characteristics are shown in Table I. The majority of patients were post-menopausal with hormone receptor-positive disease. Additionally, most patients had received different combinations of adjuvant therapy and the vast majority of patients had received adjuvant chemotherapy. Furthermore, $34 \%$ of the patients with either estrogen or progesterone receptor-positive disease had received endocrine therapy for advanced disease.

Most patients had a heavy disease burden: visceral disease was common $(82 \%)$ and liver metastases occurred in $51 \%$ of patients. Two-fifths of the patients had more than three metastatic sites. In addition, bone-only disease was observed only in five patients (Table I).

Efficacy. All 65 patients were evaluated for treatment efficacy and the PFS and OS results are shown in Figures 2 and 3. In part I of treatment, 32 patients were treated with docetaxel and 33 patients with paclitaxel. A total of 38 patients (58\%) entered part II of treatment. Of these patients, the majority had hormone receptor-positive disease (87\%) and only five patients had hormone receptor-negative disease. All hormone receptorpositive patients received endocrine therapy in part II in addition to bevacizumab according to the physician's choice, with letrozole being the most common drug $(n=19)$. Other hormonal drugs that were used included anastrozole $(n=4)$, exemestane $(n=4)$, tamoxifen $(n=3)$ and fulvestrant $(n=3)$.

The median PFS for the first-line treatment was 11.3 months (95\% CI=9.7-16.0, Figure 2) and the median OS was 35.1 months (95\% CI=22.2-50.3; Figure 3). The overall response rate was high. One patient (1.5\%) had a CR and 39 had PR $(60.0 \%)$ in part I. SD was observed in 15 patients $(23.1 \%)$. Thus, the clinical benefit rate $(\mathrm{CR}+\mathrm{PR}+\mathrm{SD})$ for part I was 84.6\%. Only three patients (4.6\%) had PD as the best response 
Table I. Demographic and baseline characteristics of patients $(n=65)$.

\begin{tabular}{|c|c|}
\hline Characteristic & Value \\
\hline Median age (range), years & $57(32-75)$ \\
\hline \multicolumn{2}{|l|}{ Menopausal status, n (\%) } \\
\hline Pre-menopausal & $10(15.4)$ \\
\hline Post-menopausal & $55(84.6)$ \\
\hline \multicolumn{2}{|c|}{ History of early-stage disease, $\mathrm{n}(\%)$} \\
\hline Total & $57(87.7)$ \\
\hline \multicolumn{2}{|l|}{ Disease-free interval, n (\%) } \\
\hline$\leq 24$ Months & $11(16.9)$ \\
\hline$>24$ Months & $46(70.8)$ \\
\hline \multicolumn{2}{|l|}{ Hormone receptor status, $\mathrm{n}(\%)$} \\
\hline $\mathrm{ER}+\mathrm{PR}+/ \mathrm{ER}+\mathrm{PR}-$ & $53(81.5)$ \\
\hline ER-PR- & $12(18.5)$ \\
\hline \multicolumn{2}{|l|}{ Estrogen receptor status, n (\%) } \\
\hline Positive & $51(78.5)$ \\
\hline Negative & $14(21.5)$ \\
\hline \multicolumn{2}{|l|}{ Progesterone receptor status, n (\%) } \\
\hline Positive & $46(70.8)$ \\
\hline Negative & $19(29.2)$ \\
\hline \multicolumn{2}{|c|}{ Prior adjuvant chemotherapy, n (\%) } \\
\hline Total & $46(70.8)$ \\
\hline Taxane & $26(40.0)$ \\
\hline Anthracycline & $38(58.5)$ \\
\hline \multicolumn{2}{|l|}{ Prior hormonal therapy, $\mathrm{n}(\%)$} \\
\hline Total & $44(67.7)$ \\
\hline (Neo)adjuvant & $38(58.5)$ \\
\hline Metastatic/advanced disease & $18(27.7)$ \\
\hline \multicolumn{2}{|l|}{ Current stage of disease, $\mathrm{n}(\%)$} \\
\hline IV & $65(100.0)$ \\
\hline \multicolumn{2}{|c|}{ Hormonal therapies used in metastatic setting, $\mathrm{n}(\%)$} \\
\hline Anastrozole & $4(10.5)$ \\
\hline Exemestane & $7(18.4)$ \\
\hline Fulvestrant & $5(13.2)$ \\
\hline Letrozole & $12(31.6)$ \\
\hline GnRH analogs & $3(7.9)$ \\
\hline Tamoxifen & $4(10.5)$ \\
\hline \multicolumn{2}{|c|}{ Number of metastatic lesions, $\mathrm{n}(\%)$} \\
\hline$\leq 3$ & $14(21.5)$ \\
\hline$>3$ & $51(78.5)$ \\
\hline \multicolumn{2}{|l|}{ Extent of disease } \\
\hline$<3$ Sites & $39(60.0)$ \\
\hline$\geq 3$ Sites & $26(40.0)$ \\
\hline \multicolumn{2}{|l|}{ Site of metastatic disease, $\mathrm{n}(\%)$} \\
\hline Visceral & $53(81.5)$ \\
\hline Non-visceral & $12(18.5)$ \\
\hline
\end{tabular}

ER: Estrogen receptor; PR: progesterone receptor; GnRH: gonadotrophin-releasing hormone.

in part I. Docetaxel- and paclitaxel-based regimens led to similar median survival values: median PFS 11.3 months $(95 \%$ CI=9.1-16.8) for docetaxel vs. 11.3 months (95\% CI 7.4-30.7, $p=0.47$ ) for paclitaxel, median OS 38 months (95\% CI=19.850.4) vs. 34.2 months (95\% CI=18.1-not reached, $p=0.77$ ) respectively. The median OS for patients with hormone receptor-positive disease was 45.0 months (95\% CI=30.2-51.3) and for patients with triple-negative disease, it was 17.9 months (95\% CI=8.5-26.9, $p=0.011)$.

Subsequent therapy. Patients were allowed to receive bevacizumab together with a second-line chemotherapy according to investigators' choice. A total of 17 patients began second-line bevacizumab-chemotherapy combination. The preferred chemotherapy in the protocol was capecitabine $(n=15)$ but patients also received paclitaxel and vinorelbine. The median PFS for second-line therapy was 5.1 months $(95 \%$ $\mathrm{CI}=4.4-16.1$ months) and the OS was 33.8 months $(95 \%$ $\mathrm{CI}=24.7$ months-NR). With the second-line bevacizumabchemotherapy, seven patients responded partially (41\%) and six patients had SD as the best response to the treatment (35\%). No CRs were observed. Disease progression occurred in three patients $(18 \%)$. For one patient, the response could not be defined because at data closure, the first response evaluation had not yet been performed.

Safety. During part I of the treatment, the bevacizumabchemotherapy combination was generally well tolerated and most toxicity was mild (grade 1-2). The worst grade of a side-effect per patient is presented. The adverse events of all grades (1-4) that were most frequently reported were neutropenia $(n=45,69 \%)$, musculoskeletal pain $(n=45,69 \%)$, alopecia $(n=44,68 \%)$, leukocytopenia $(n=41,63 \%)$, fatigue $(n=35,54 \%)$, mucositis $(n=35,54 \%)$, anemia $(n=35,54 \%)$, epistaxis $(n=34,52 \%)$, constipation $(n=27,42 \%)$, nail disorders $(n=23,35 \%)$, proteinuria $(n=22,34 \%)$, diarrhea $(n=22,34 \%)$, elevated liver enzymes $(n=20,31 \%)$, nausea $(n=20,31 \%)$ and peripheral neuropathy $(n=18,28 \%)$. Serious adverse events during part I chemotherapy treatment are presented in Table II. The most common serious adverse event was neutropenia but febrile neutropenia was rare. One patient had a grade 5 toxicity due to the treatment and died during part I of the study. This patient had pre-existing diverticulosis and then developed diverticulitis, which resulted in gastrointestinal perforation and peritonitis. During bevacizumab maintenance, grade 3-4 adverse events were rare. The serious adverse events from part II treatment are presented in Table III.

Bevacizumab treatment-related adverse events according to the investigators' judgment are summarized in Table IV. The gastrointestinal perforation, mentioned above, was suspected to be related to bevacizumab. Hypertension and proteinuria were frequently reported but were usually of low grade. However, one patient suffered from grade 4 proteinuria and renal failure. In addition, over half of the patients had low-grade epistaxis.

In the second-line setting, the expected side-effects for capecitabine occurred in 17 patients treated in this part of the trial. The serious adverse events reported were grade 3 hand and foot syndrome $(n=3)$ and a single case of grade 4 diarrhea. 


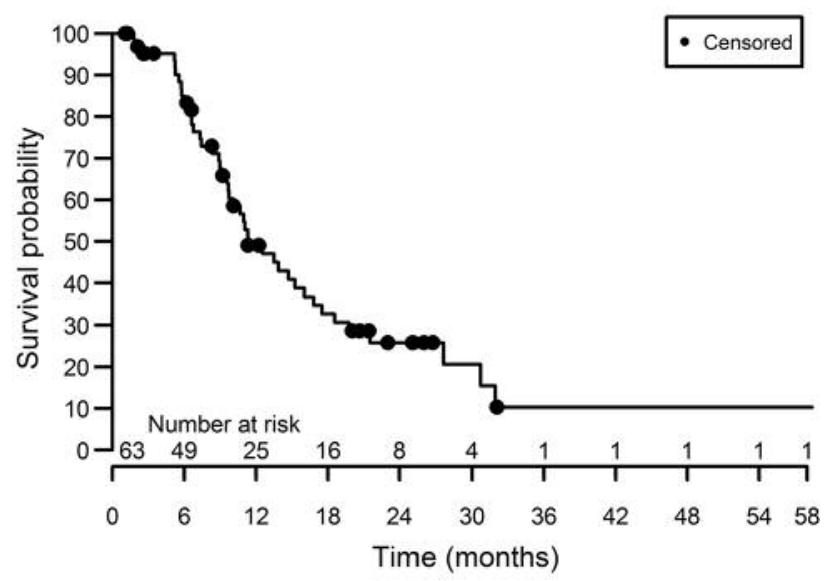

Figure 2. Progression-free survival for the whole patient cohort.

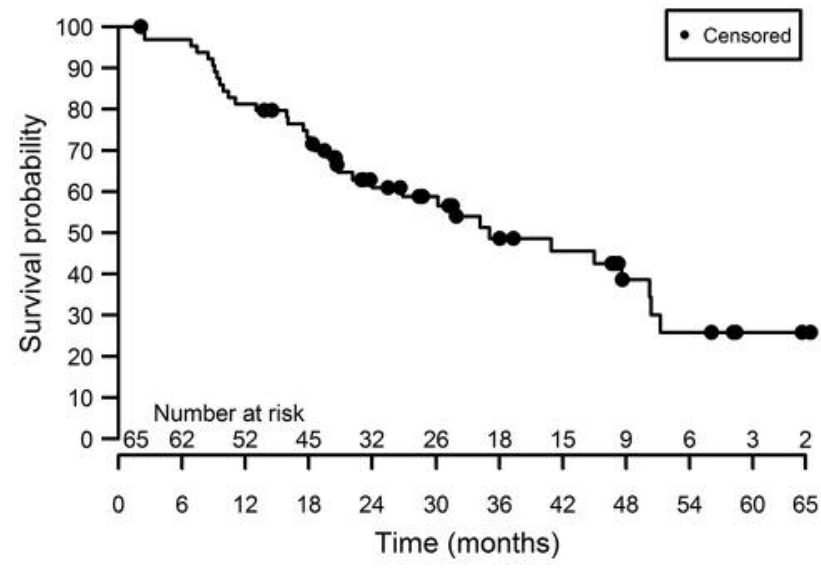

Figure 3. Overall survival for the whole patient cohort.

\section{Discussion}

This study resulted in excellent OS of almost 3 years (35.1 months) in patients with advanced breast cancer with poor prognostic features at the beginning of the trial. Visceral metastases were common (80\%), and most patients had multiple metastases. Prior taxane treatment as adjuvant chemotherapy was given to $40 \%$ of these patients. The most favorable results towards a benefit from adding bevacizumab to chemotherapy are reported in the E2100 trial (13). In that study, the number of patients with visceral disease was similar to that observed in our study (79.5-87.1\% depending on the treatment arm). Additionally, the extent of disease (42.0-46.3\% of patients had more than three metastatic sites) was quite similar in both studies. Only approximately $15 \%$ of E2100 patients were pre-treated with taxanes in an adjuvant setting compared to $40 \%$ of our patients. PFS was reported
Table II. Grade 3-4 adverse events experienced by patients in part I of the treatment.

\begin{tabular}{lcc}
\hline & \multicolumn{2}{c}{ Patients (n=65) } \\
\cline { 2 - 3 } Adverse event & Grade 3 & Grade 4 \\
\hline Fatigue & 2 & \\
Neutropenia & 9 & 16 \\
Leukocytopenia & 11 & 2 \\
Elevated liver enzymes & 1 & 1 \\
Infection & 9 & \\
Febrile neutropenia or neutropenic sepsis & 3 & 1 \\
Peripheral neuropathy & 1 & \\
Pain & 3 & 1 \\
Diarrhea & 1 & \\
Nausea & 1 & \\
Cardiac disorders & $1 *$ & \\
Osteonecrosis of the jaw & 1 & \\
Drug hypersensitivity & 1 & \\
Gastrointestinal perforation & & $1 * *$ \\
\hline
\end{tabular}

*Supraventricular tachycardia; **patient died, grade 5 adverse event.

Table III. Grade 3-4 adverse events experienced by patients in part II of the treatment.

\begin{tabular}{lcc}
\hline & \multicolumn{2}{c}{ Grade 3-4/patients } \\
\cline { 2 - 3 } Adverse event & $\mathrm{HR}+(\mathrm{n}=33)$ & $\mathrm{HR}-(\mathrm{n}=5)$ \\
\hline Infection & 2 \\
Leukocytopenia & 1 \\
Elevated liver enzymes & 2 & \\
Peripheral neuropathy & 1 & \\
Anorexia & 1 & 1 \\
Cardiac disorders & $2^{*}$ & \\
Hyponatremia & 1 & \\
\hline
\end{tabular}

HR+: Hormone receptor-positive (estrogen receptor+ or progesterone receptor+); HR-: hormone receptor-negative. *Congestive heart failure, coronary artery thrombosis.

Table IV. Bevacizumab-related events experienced by patients in this study.

Patients $(n=65)$

Adverse event

Grade 1-2 Grade 3 Grade 4 Grade 5

Hypertension

Proteinuria

Bleeding/hemorrhage

Epistaxis

Gastrointestinal fistula/abscess

Gastrointestinal perforation 
to be very similar between the E2100 study and our study (11.8 months in E2100 and 11.3 months in our trial). Nevertheless, the OS was remarkably longer in our trial: 35.1 months compared to 26.7 months observed in the E2100 trial.

There are some possible explanations for the long OS observed in this study. The main difference in our study when compared to other studies of first-line chemotherapy combining bevacizumab with taxanes $(13,14,16,18)$ is that after a maximal response was reached in our study, bevacizumab was continued as a maintenance treatment with endocrine therapy in patients with hormone receptor-positive disease. Bevacizumab maintenance was given to 38 patients $(58 \%)$ and the majority of these patients $(87 \%)$ had hormone receptor-positive disease and received endocrine therapy with bevacizumab. Intracellular estrogen signaling pathways and VEGF pathways have several interactions $(19,20,24,25)$; therefore, endocrine treatment may add a substantial benefit to bevacizumab monotherapy, as also recently shown with androgen signaling pathways and VEGF in prostate cancer (26). In addition, using biweekly instead of triweekly docetaxel infusions might have led to lower treatment toxicity and, therefore, to prolonged survival. This was previously demonstrated in our randomized phase III Prosty trial where triweekly and biweekly docetaxel dosing were compared in advanced castration-resistant prostate cancer (27). Weekly paclitaxel compared to triweekly infusions has also demonstrated survival benefit in advanced breast cancer (28).

This trial has many differences compared to the LEA trial (21). In the LEA trial, patients with advanced disease were endocrine treatment-naïve. In our study, one-third of the patients with hormone receptor-positive disease had received hormonal therapies for advanced disease, meaning that the patients seemed to have less hormone treatment-sensitive disease. Half of the patients in our trial also had liver metastasis compared to only $20 \%$ in the LEA trial. Thus, our patients had less favorable prognoses. The OS for this patient population is, as expected, shorter with less favorable prognostic features. In the LEA trial, the OS was 52.1 months in patients treated with the first-line bevacizumabendocrine therapy combination. This exceeds that of the patients with hormone receptor-positive disease of our study by only 7.1 months, which is less than expected considering the poor prognostic features of the disease at the beginning of our patients' treatments. Both these studies favor the hypothesis of an interaction between hormonal and angiogenetic cellular pathways in breast cancer.

In preclinical studies, it has been reported that tumor progression may be accelerated after short-term angiogenesis inhibition (29). On the other hand, treating colorectal cancer with second-line bevacizumab-chemotherapy combination after disease progression with first-line therapy including bevacizumab was shown to have survival benefits (30). Therefore, some patients with metastatic adenocarcinoma may benefit from prolonged VEGF inhibition in terms of survival. This is one possible explanation for the long OS seen in our study.

High response rates have been reported in all of the trials with bevacizumab combined with a first-line chemotherapeutic. The response rates previously reported with the bevacizumabtaxane combination range from $36.9 \%$ to $64.1 \%$ compared to $21.2-46.4 \%$ with single taxane therapy $(13,14,31)$. Similarly, good responses were achieved in this study. The clinical benefit rate was $84.6 \%$ and $62 \%$ of patients responded at least with PR according to the RECIST criteria, which is in line with previously published data.

In this small series of patients, no difference in PFS or OS was observed between the two taxane-treated groups. Half of the patients in the study were treated with paclitaxel and the other half with docetaxel. Thus, there is an indication that docetaxel and paclitaxel are similarly effective with bevacizumab.

Bevacizumab adds treatment toxicity compared to single taxane chemotherapy. In this study, bevacizumab-related serious events were rare (10.8\%). However, one patient died because of bevacizumab-related toxicity, which in this case was a fatal peritonitis. The contributing factor was underlying diverticulosis in our patient. Additionally, high-grade proteinuria and hypertension were observed, which are known side-effects of bevacizumab (13-17, 31). Caution should be exercised when treating patients with known risk factors for the use of bevacizumab, namely a history of thromboembolic events, cardiovascular disease or risk factors for abdominal infection and fistula, among others. The other grade 3-4 toxicities observed were related to chemotherapy or to the metastatic disease itself and were reported at the anticipated rates. In the AVADO trial, $75-78 \%$ of the patients, depending on the treatment arm, treated with a bevacizumabdocetaxel combination had at least one grade 3 toxicity due to the treatment (14), whereas a minimum of grade 3 toxicity was observed in $71 \%$ of our patients. Only $24 \%$ of the patients had grade 3-4 toxicity during bevacizumabcapecitabine treatment in our study. No unexpected new sideeffects were reported in our study. In conclusion, combining bevacizumab with paclitaxel or docetaxel or to second-line capecitabine has an acceptable side-effect profile.

The small sample size does not allow us to draw any conclusions about the efficacy of second-line chemotherapy.

Although our patients presented many poor prognostic features at baseline, the OS achieved of nearly 3 years is remarkable. This study intended to determine whether bevacizumab adds an advantage to taxane treatment followed by a bevacizumab maintenance therapy with an endocrine therapy. With an OS of 17.9 months in patients with triplenegative disease and 45.0 months in a hormone receptorpositive study population, it can be concluded that combining bevacizumab with a conventional taxane 
treatment is a treatment option. This is especially true in patients with a heavy disease burden and needing rapid tumor shrinkage. We have gathered a comprehensive serum, plasma and tumor biopsy collection from the study population and we aim to explore markers predictive for the long response to bevacizumab combination therapies.

\section{Acknowledgements}

The Authors would like to thank the research nurses and the patients that participated in this study. Roche Inc., Basel, Swiss supported the study financially (study monitoring, electronic CRF-system and partially expenses of bevacizumab).

\section{References}

1 Andre F, Slimane K, Bachelot T, Dunant A, Namer M, Barrelier A, Kabbaj O, Spano JP, Marsiglia H, Rouzier R, Delaloge S and Spielmann M: Breast cancer with synchronous metastases: trends in survival during a 14-year period. J Clin Oncol 22: 3302-3308, 2004.

2 Foukakis T, Fornander T, Lekberg T, Hellborg H, Adolfsson J and Bergh J: Age-specific trends of survival in metastatic breast cancer: 26 years longitudinal data from a population-based cancer registry in Stockholm, Sweden. Breast Cancer Res Treat 130: 553-560, 2011.

3 Finnish cancer registry. (http://www.cancerregistry.fi) Updated March 3, 2016. Assessed September 21, 2016.

4 Allemani C, Weir HK, Carreira H, Harewood R, Spika D, Wang XS, Bannon F, Ahn JV, Johnson CJ, Bonaventure A, MarcosGragera R, Stiller C, Azevedo e Silva G, Chen WQ, Ogunbiyi OJ, Rachet B, Soeberg MJ, You H, Matsuda T, Bielska-Lasota M, Storm H, Tucker TC, Coleman MP and CONCORD Working Group: Global surveillance of cancer survival 1995-2009: analysis of individual data for $25,676,887$ patients from 279 population-based registries in 67 countries (CONCORD-2). Lancet 385: 977-1010, 2015.

5 Cardoso F, Costa A, Norton L, Senkus E, Aapro M, Andre F, Barrios CH, Bergh J, Biganzoli L, Blackwell KL, Cardoso MJ, Cufer T, El Saghir N, Fallowfield L, Fenech D, Francis P, Gelmon K, Giordano SH, Gligorov J, Goldhirsch A, Harbeck N, Houssami N, Hudis C, Kaufman B, Krop I, Kyriakides S, Lin UN, Mayer M, Merjaver SD, Nordstrom EB, Pagani O, Partridge A, PenaultLlorca F, Piccart MJ, Rugo H, Sledge G, Thomssen C, Van't Veer L, Vorobiof D, Vrieling C, West N, Xu B and Winer E: ESOESMO 2nd international consensus guidelines for advanced breast cancer (ABC2)dagger. Ann Oncol 25: 1871-1888, 2014.

6 Cortes J, O'Shaughnessy J, Loesch D, Blum JL, Vahdat LT, Petrakova K, Chollet P, Manikas A, Dieras V, Delozier T, Vladimirov V, Cardoso F, Koh H, Bougnoux P, Dutcus CE, Seegobin S, Mir D, Meneses N, Wanders J, Twelves C and EMBRACE (Eisai Metastatic Breast Cancer Study Assessing Physician's Choice Versus E7389) investigators: Eribulin monotherapy versus treatment of physician's choice in patients with metastatic breast cancer (EMBRACE): a phase 3 open-label randomised study. Lancet 377: 914-923, 2011.

7 Marty M and Pivot X: The potential of anti-vascular endothelial growth factor therapy in metastatic breast cancer: clinical experience with anti-angiogenic agents, focusing on bevacizumab. Eur J Cancer 44: 912-920, 2008.

8 Chen YX, Yang Q, Kuang JJ, Chen SY, Wei Y, Jiang ZM and Xie DR: Efficacy of adding bevacizumab in the first-line chemotherapy of metastatic colorectal cancer: evidence from seven randomized clinical trials. Gastroenterol Res Pract 2014: 594930, 2014.

9 Sandler A, Gray R, Perry MC, Brahmer J, Schiller JH, Dowlati A, Lilenbaum R and Johnson DH: Paclitaxel-carboplatin alone or with bevacizumab for non-small-cell lung cancer. N Engl J Med 355: 2542-2550, 2006.

10 Bracarda S, Bellmunt J, Melichar B, Negrier S, Bajetta E, Ravaud A, Sneller V and Escudier B: Overall survival in patients with metastatic renal cell carcinoma initially treated with bevacizumab plus interferon-alpha2a and subsequent therapy with tyrosine kinase inhibitors: a retrospective analysis of the phase III AVOREN trial. BJU Int 107: 214-219, 2011.

11 Gaitskell K, Martinek I, Bryant A, Kehoe S, Nicum S and Morrison $\mathrm{J}$ : Angiogenesis inhibitors for the treatment of ovarian cancer. Cochrane Database Syst Rev (9):CD007930. doi: CD007930, 2011.

12 NCCN Clinical Practice guidelines in oncology. Breast Cancer. (http://www.ncen.org) Version 2.2016. Assessed September 21, 2016.

13 Miller K, Wang M, Gralow J, Dickler M, Cobleigh M, Perez EA, Shenkier T, Cella D and Davidson NE: Paclitaxel plus bevacizumab versus paclitaxel alone for metastatic breast cancer. N Eng1 J Med 357: 2666-2676, 2007.

14 Miles DW, Chan A, Dirix LY, Cortes J, Pivot X, Tomczak P, Delozier T, Sohn JH, Provencher L, Puglisi F, Harbeck N, Steger GG, Schneeweiss A, Wardley AM, Chlistalla A and Romieu G: Phase III study of bevacizumab plus docetaxel compared with placebo plus docetaxel for the first-line treatment of human epidermal growth factor receptor 2-negative metastatic breast cancer. J Clin Oncol 28: 3239-3247, 2010.

15 Miller KD, Chap LI, Holmes FA, Cobleigh MA, Marcom PK, Fehrenbacher L, Dickler M, Overmoyer BA, Reimann JD, Sing AP, Langmuir V and Rugo HS: Randomized phase III trial of capecitabine compared with bevacizumab plus capecitabine in patients with previously treated metastatic breast cancer. J Clin Oncol 23: 792-799, 2005.

16 Robert NJ, Dieras V, Glaspy J, Brufsky AM, Bondarenko I, Lipatov ON, Perez EA, Yardley DA, Chan SY, Zhou X, Phan SC and O'Shaughnessy J: RIBBON-1: randomized, doubleblind, placebo-controlled, phase III trial of chemotherapy with or without bevacizumab for first-line treatment of human epidermal growth factor receptor 2-negative, locally recurrent or metastatic breast cancer. J Clin Oncol 29: 1252-1260, 2011.

17 Brufsky AM, Hurvitz S, Perez E, Swamy R, Valero V, O’Neill V and Rugo HS: RIBBON-2: a randomized, double-blind, placebocontrolled, phase III trial evaluating the efficacy and safety of bevacizumab in combination with chemotherapy for second-line treatment of human epidermal growth factor receptor 2-negative metastatic breast cancer. J Clin Oncol 29: 4286-4293, 2011.

18 Zielinski C, Lang I, Inbar M, Kahan Z, Greil R, Beslija S, Stemmer SM, Zvirbule Z, Steger GG, Melichar B, Pienkowski T, Sirbu D, Petruzelka L, Eniu A, Nisenbaum B, Dank M, Anghel R, Messinger D, Brodowicz $\mathrm{T}$ and TURANDOT investigators: Bevacizumab plus paclitaxel versus bevacizumab plus capecitabine as first-line treatment for HER2-negative metastatic breast cancer (TURANDOT): primary endpoint results of a randomised, openlabel, non-inferiority, phase 3 trial. Lancet Oncol, 2016. 
19 Roy V and Perez EA: Biologic therapy of breast cancer: focus on co-inhibition of endocrine and angiogenesis pathways. Breast Cancer Res Treat 116: 31-38, 2009.

20 Manders P, Beex LV, Tjan-Heijnen VC, Span PN and Sweep CG: Vascular endothelial growth factor is associated with the efficacy of endocrine therapy in patients with advanced breast carcinoma. Cancer 98: 2125-2132, 2003.

21 Martin M, Loibl S, von Minckwitz G, Morales S, Martinez N, Guerrero A, Anton A, Aktas B, Schoenegg W, Munoz M, GarciaSaenz JA, Gil M, Ramos M, Margeli M, Carrasco E, Liedtke C, Wachsmann G, Mehta K and De la Haba-Rodriguez JR: Phase III trial evaluating the addition of bevacizumab to endocrine therapy as first-line treatment for advanced breast cancer: the letrozole/fulvestrant and avastin (LEA) study. J Clin Oncol 33: 1045-1052, 2015.

22 Cancer Therapy Evaluation Program, Common Terminology Criteria for Adverse Events, Version 3.0, DCTD, NCI, NIH, DHHS. (http://ctep.cancer.gov). Publish Date: August 9, 2006.

23 Eisenhauer EA, Therasse P, Bogaerts J, Schwartz LH, Sargent D, Ford R, Dancey J, Arbuck S, Gwyther S, Mooney M, Rubinstein L, Shankar L, Dodd L, Kaplan R, Lacombe D and Verweij J: New response evaluation criteria in solid tumours: revised RECIST guideline (version 1.1). Eur J Cancer 45: 228-247, 2009.

24 Gagliardi AR, Hennig B and Collins DC: Antiestrogens inhibit endothelial cell growth stimulated by angiogenic growth factors. Anticancer Res 16: 1101-1106, 1996.

25 Takei H, Lee ES and Jordan VC: In vitro regulation of vascular endothelial growth factor by estrogens and antiestrogens in estrogen-receptor positive breast cancer. Breast Cancer 9: 39-42, 2002.

26 McKay RR, Zurita AJ, Werner L, Bruce JY, Carducci MA, Stein MN, Heath EI, Hussain A, Tran HT, Sweeney CJ, Ross RW, Kantoff PW, Slovin SF and Taplin ME: A Randomized Phase II Trial of Short-Course Androgen Deprivation Therapy With or Without Bevacizumab for Patients With Recurrent Prostate Cancer After Definitive Local Therapy. J Clin Oncol 34: 19131920, 2016.
27 Kellokumpu-Lehtinen PL, Harmenberg U, Joensuu T, McDermott R, Hervonen P, Ginman C, Luukkaa M, Nyandoto P, Hemminki A, Nilsson S, McCaffrey J, Asola R, TurpeenniemiHujanen T, Laestadius F, Tasmuth T, Sandberg K, Keane M, Lehtinen I, Luukkaala T, Joensuu H and PROSTY study group: 2-Weekly versus 3-weekly docetaxel to treat castration-resistant advanced prostate cancer: a randomised, phase 3 trial. Lancet Oncol 14: 117-124, 2013.

28 Mauri D, Kamposioras K, Tsali L, Bristianou M, Valachis A, Karathanasi I, Georgiou C and Polyzos NP: Overall survival benefit for weekly $v s$. three-weekly taxanes regimens in advanced breast cancer: A meta-analysis. Cancer Treat Rev 36: 69-74, 2010.

29 Ebos JM, Lee CR, Cruz-Munoz W, Bjarnason GA, Christensen JG and Kerbel RS: Accelerated metastasis after short-term treatment with a potent inhibitor of tumor angiogenesis. Cancer Cell 15: 232-239, 2009.

30 Bennouna J, Sastre J, Arnold D, Osterlund P, Greil R, Van Cutsem E, von Moos R, Vieitez JM, Bouche O, Borg C, Steffens CC, Alonso-Orduna V, Schlichting C, Reyes-Rivera I, Bendahmane B, Andre T, Kubicka S and ML18147 Study Investigators: Continuation of bevacizumab after first progression in metastatic colorectal cancer (ML18147): a randomised phase 3 trial. Lancet Oncol 14: 29-37, 2013.

31 Lang I, Brodowicz T, Ryvo L, Kahan Z, Greil R, Beslija S, Stemmer SM, Kaufman B, Zvirbule Z, Steger GG, Melichar B, Pienkowski T, Sirbu D, Messinger D, Zielinski C and Central European Cooperative Oncology Group: Bevacizumab plus paclitaxel versus bevacizumab plus capecitabine as first-line treatment for HER2-negative metastatic breast cancer: interim efficacy results of the randomised, open-label, non-inferiority, phase 3 TURANDOT trial. Lancet Oncol 14: 125-133, 2013.

Received September 28, 2016

Revised October 21, 2016

Accepted October 26, 2016 